Research Article

\title{
Significance of MHD Radiative Non-Newtonian Nanofluid Flow towards a Porous Channel: A Framework of the Casson Fluid Model
}

\author{
N. Thamaraikannan $\mathbb{D}^{1},{ }^{1}$ S. Karthikeyan, ${ }^{1}$ and Dinesh Kumar Chaudhary ${ }^{2}{ }^{2}$ \\ ${ }^{1}$ Department of Mathematics, Erode Arts and Science College, Erode 638 009, Tamilnadu, India \\ ${ }^{2}$ Department of Physics, Amrit Campus, Tribhuvan University, Kathmandu, Nepal \\ Correspondence should be addressed to N. Thamaraikannan; kthamkar@gmail.com and Dinesh Kumar Chaudhary; din.2033@ \\ gmail.com
}

Received 1 April 2021; Accepted 18 May 2021; Published 7 June 2021

Academic Editor: Riaz Ahmad

Copyright (c) 2021 N. Thamaraikannan et al. This is an open access article distributed under the Creative Commons Attribution License, which permits unrestricted use, distribution, and reproduction in any medium, provided the original work is properly cited.

\begin{abstract}
The effect of various parameters in the unsteady pulsating flow of radiative hydromagnetic Casson nanofluid through a porous channel is investigated. The governing equations were nondimensionalized by applying suitable transformations. The perturbation technique was employed to solve the resulting similarity equations. The velocity and temperature fields are illustrated for several pertinent flow parameters. The fluid velocity has been enhancing for higher values of the frequency parameter, Casson fluid parameter, nanoparticle volume fraction, and Darcy number. The reverse impact is observed for larger values of the Hartmann number. The result reveals that adding the nanoparticles has enhanced the heat transfer of the base fluid as the nanoparticles increase the heat conductivity. Furthermore, it is noticed that the temperature profile decreases rapidly for higher values of the cross Reynolds number and the radiation parameter. Finally, an excellent agreement between the current results and previous results is obtained by comparing with the available limiting results in the literature.
\end{abstract}

\section{Introduction}

Nanofluid consists of the uniform composition of solid particles whose dimension is in the range of 1 to $100 \mathrm{~nm}$. The heat transfer characteristics of nanofluid will be controlled by low thermal conductivity. Due to this constraint, the heat transfer ability of conventional heat transfer fluids such as water, lubricant oil, and ethylene glycol has been highly limited since they provided low thermal conductivity. Generally, metals exhibit higher thermal conductivity than typical heat transfer fluids. Mixing low-dimension metallic particles (nanoparticles) with fluids will help increase the thermal conductivity. This process is identified as an innovative technique for enhancing the heat transfer coefficient. Choi [1] attempted this first and succeeded. Nisar et al. [2] investigated the steady flow with heat diffusion of blood which transmits the micropolar nanoliquid with gold particles through a curved shrinking/stretched surface with the impact of radiation. They suggested that nanoparticlecontaining gold can treat cancer as these materials have a lofty atomic quantity that produces the temperature guide to the handling of malignant tumours. The heat transfer behaviors in the presence of the magnetic field were investigated using the finite-element method by Sheikholeslami et al. [3]. Dogonchi et al. [4] examined the magnetohydrodynamic (MHD) flow of nanofluid and heat transmission between two surfaces by taking into account the Joule heat effects. Their study has identified an increase in the concentration, Nusselt number, and temperature profiles with an increase in the magnitude of the Schmidt number. Magnetohydrodynamics (MHD) is a branch of dynamics which deals with fluids during electrical conduction, such as electrolytes, plasmas, and liquid metals. The primary principle of MHD is to produce forces in the fluid when the 
magnetic field induces a current through a moving conducting fluid. Magnetohydrodynamics has been found in diverse engineering applications. Some other cutting-edge research reports about the nanofluid features and applications are given in [5-8].

The Casson fluid was first introduced in 1959 by Casson. He used pigment oil of printing ink to scrutinize the flow behaviour. The "Casson fluid" is noted as the most popular non-Newtonian fluid. It behaves like a Newtonian fluid above a critical stress value, and at low shear strain, it behaves like an elastic solid. The Casson fluid model can be chosen for analyzing the rheological behaviour of the ingredients of fluids such as soup, honey, coolant oil, and human blood. Abolbashari et al. [9] analyzed the flow, mass, and heat transfer characteristics of the Casson nanofluid over a stretching surface analytically. Sheikh et al. [10] investigated "the MHD free convection flow of the Casson fluid in a channel," and they found that "the larger values of the Casson parameter elevate the velocity of the Casson fluid." The rate of heat transfer of the steady MHD stagnation point flow of the Casson fluid on the shrinking/ stretching surface with the effect of thermal radiation and viscous dissipation was investigated by Lund et al. [11]. Tassaddiq et al. [12] analyzed the Newtonian heating effects for the generalized Casson fluid MHD flow with porous effects. They found that velocity grows with an increase in the porosity parameter, and the fluid flow is controllable for all possible values of the Casson parameter. The magnetohydrodynamic boundary layer flow of the Casson fluid over an exponentially permeable shrinking sheet was investigated by Nadeem et al. [13].

Thermal radiation plays a vital role in many industrial processes. Kumar et al. [14] applied the Buongiorno model to investigate the pulsatile flow of the MHD Casson nanofluid through a vertical porous channel with thermal radiation. MHD flow through a porous channel between two parallel plates has enormous applications such as in petrochemical industries, power generators, polymer technology, and food industries. Falede et al. [15] investigated "MHD oscillatory flow through a porous channel saturated with porous media." "MHD flow through a vertical channel with a porous medium" was analyzed by Dwivedi et al. [16]. Loganathan et al. [17] investigated the entropy features of a MHD third-grade nanoliquid induced by a linearly stretching plate. They found that the Bejan number rate is enhanced due to thermal radiation effects. Srinivas et al. [18] studied the effect of the applied magnetic field, chemical reaction, and thermal radiation on the pulsating flow of the Casson fluid in a porous channel. Anwar et al. [19] explained the transient free convection phenomenon in a vertical porous channel subject to nonlinear thermal radiation. The important visualization about thermal radiation with various flow geometries is found in the literature [20-25].

To this extent, our literature review confirms that there are not existing papers for hydromagnetic pulsating Casson nanofluid flow through a porous channel with radiation effects. An examination was made for pulsating Casson fluid flow through a porous channel with radiation and chemical reaction by Srinivas et al. [18]. However, similar research for the Casson nanofluid with brief discussions is still limited. Therefore, we expanded our effort to the pulsative Casson nanofluid that passes through a porous channel with radiation and magnetic-field impacts. The considered problem has enormous applications in many industrial as well as biological processes such as crude oil purification and blood flow through contracting muscles. The perturbation technique was employed to solve the resulting similarity equations. As a method for finding analytical solutions approximately, the perturbation method is an exciting tool among other analytical methods. Due to its unfussiness and high precision, the perturbation method was applied for solving many heat transfer and fluid mechanics problems. The solution for some fluid flow problems can be found in [26-29].

\section{Mathematical Formulation}

We consider the influence of uniform traverse applied magnetic field on the pulsatile incompressible flow of the Casson nanofluid in a porous channel. The flow is driven by an unsteady pressure gradient [18].

$$
\frac{1}{\rho_{\mathrm{nf}}} \frac{\partial p^{*}}{\partial x^{*}}=-A\left\{1+\varepsilon e^{i \omega t^{*}}\right\},
$$

where $\rho_{\mathrm{nf}}$ is the density of the nanofluid, $p^{*}$ is the pressure, $\varepsilon \ll 1$ is the suitably chosen positive quantity, " $A$ " is a constant, and $\omega$ is the frequency.

As in Figure 1, the $X^{*}$-axis is taken along the lower wall, and the perpendicular axis is noted as the $Y^{*}$-axis. The lower wall maintains the temperature $T_{0}$, and the upper wall maintains the temperature $T_{1}\left(T_{0}<T_{1}\right)$. The nanofluid is injected from the lower wall into the porous channel with the velocity $v_{0}$, and it is sucked out from the porous medium via the upper wall with a similar velocity. Uniform magnetic field $\left(B_{0}\right)$ is imposed along the usual direction to the flow. The physical flow system is noted in Figure 1.

The Casson nanofluid's rheological equation is stated ast

$$
T_{i j}=\left\{\begin{array}{l}
\left(2 \mu_{\mathrm{Bnf}}+\frac{p_{y}}{\sqrt{\left(\pi_{c} / 2\right)}}\right) e_{m n}, \pi_{c}>\pi, \\
\left(2 \mu_{\mathrm{Bnf}}+\frac{p_{y}}{\sqrt{\left(\pi_{c} / 2\right)}}\right) e_{m n}, \pi_{c}<\pi,
\end{array}\right.
$$

where " $\pi_{c}$ " is the critical value of " $\pi$ " based on the nonNewtonian model. $T_{m n}$ is the $(m, n)^{\text {th }}$ stress tensor component, and $\pi=e_{m n}$, with $e_{m n}$ being the $(m, n)^{\text {th }}$ deformation rate. $\mu_{\mathrm{Bnf}}$ is the plastic "dynamic viscosity" of the "non-Newtonian" nanofluid. $p_{y}$ is the "yield stress" of the fluid.

The problem's governing equation is as follows: 


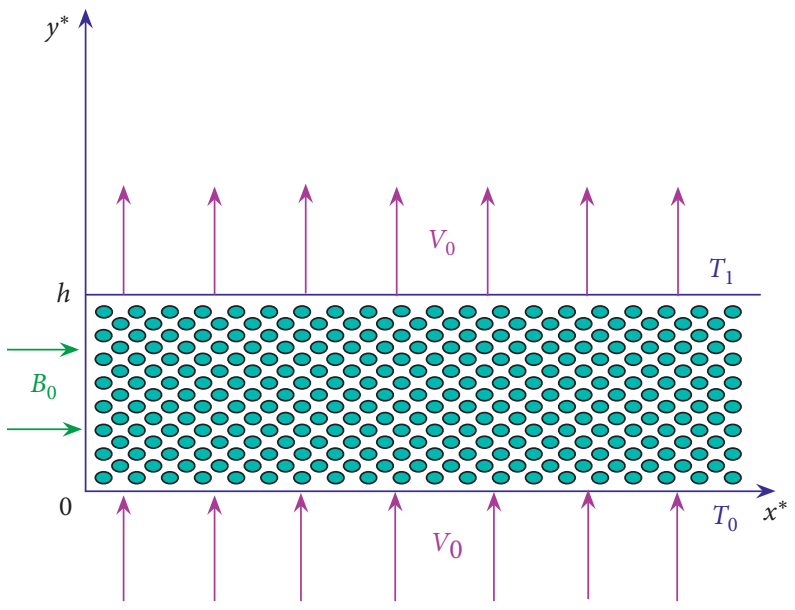

Figure 1: Physical model and the coordinate system.

$$
\begin{aligned}
\frac{\partial u^{*}}{\partial t^{*}}+v_{0} \frac{\partial u^{*}}{\partial y^{*}} & =-\frac{1}{\rho_{n f}} \frac{\partial p^{*}}{\partial x^{*}}+\frac{\mu_{n f}}{\rho_{n f}}\left(1+\frac{1}{\beta}\right) \frac{\partial^{2} u^{*}}{\partial y^{* 2}}-\frac{\sigma B_{0}^{2}}{\rho_{n f}} u^{*}-\frac{\mu_{n f} \Phi}{\rho_{n f} k} u^{*} \\
0 & =-\frac{1}{\rho_{n f}} \frac{\partial p^{*}}{\partial y^{*}}
\end{aligned}
$$

$$
\frac{\partial T^{*}}{\partial t^{*}}+v_{0} \frac{\partial T^{*}}{\partial y^{*}}=-\frac{\kappa_{n f}}{\left(\rho C_{p}\right)_{n f}} \frac{\partial^{2} T^{*}}{\partial y^{* 2}}+\frac{\mu_{n f}}{\left(\rho C_{p}\right)_{n f}}\left(1+\frac{1}{\beta}\right)\left(\frac{\partial u^{*}}{\partial y^{*}}\right)^{2}+\frac{\sigma B 0^{2}}{\left(\rho C_{p}\right)_{n f}} u^{* 2}-\frac{1}{\left(\rho C_{p}\right)_{n f}} \frac{\partial q_{r}}{\partial y^{*}}+\frac{Q_{0}}{\left(\rho C_{p}\right)_{n f}}\left(T^{*}-T_{0}\right)
$$

The boundary conditions for the present analysis are [18]

$$
\begin{array}{ll}
u^{*}=0, T^{*}=T_{0}, & \text { at } y^{*}=0, \\
u^{*}=0, T^{*}=T_{1}, & \text { at } y^{*}=L,
\end{array}
$$

where $u^{*}$ is the dimensional velocity in the $X$-direction, $k$ and $\Phi$ are the permeability and porosity of the porous medium, $\sigma$ is the electrical conductivity of the fluid, $\mu_{\mathrm{nf}}$ is the dynamic viscosity of the nanofluid, $\rho_{\mathrm{nf}}$ is the density of the nanofluid, $\nu_{\mathrm{nf}}$ is the kinematic viscosity of the Casson nanofluid, $C_{\mathrm{pnf}}$ is the specific heat at a constant pressure of the nanofluid, and $\kappa_{\mathrm{nf}}$ is the thermal conductivity of the nanofluid, which is given by Oztop and Abu-Nada as follows [14]:

$$
\begin{aligned}
\rho_{\mathrm{nf}} & =(1-\varphi) \rho_{f}+\varphi \rho_{n}, \\
\mu_{\mathrm{nf}} & =\frac{\mu_{f}}{(1-\varphi)^{2.5}}, \\
\left(\rho C_{p}\right)_{\mathrm{nf}} & =(1-\varphi)\left(\rho C_{p}\right)_{f}+\varphi\left(\rho C_{p}\right)_{n}, \\
\nu_{\mathrm{nf}} & =\frac{\mu_{\mathrm{nf}}}{\rho_{\mathrm{nf}}} \\
\frac{\kappa_{\mathrm{nf}}}{\kappa_{f}} & =\frac{\left(2 \kappa_{f}+\kappa_{n}\right)-2 \varphi\left(\kappa_{f}-\kappa_{n}\right)}{\left(2 \kappa_{f}+\kappa_{n}\right)+\varphi\left(\kappa_{f}-\kappa_{n}\right)} .
\end{aligned}
$$

Here, $\varphi$ is the nanoparticle volume fraction, $\left(\rho C_{p}\right)_{\mathrm{nf}}$ is the heat capacity of the nanofluid, and $\left(\rho C_{p}\right)_{f}$ and $\left(\rho C_{p}\right)_{n}$ are the heat capacity of the base fluid and the nanoparticles, respectively. $\rho_{f}$ and $\rho_{n}$ are the densities of the fluid and the solid fractions, respectively. $\mu_{f}, \nu_{f}$, and $C_{\mathrm{pf}}$ are the dynamic viscosity, kinematic viscosity, and specific heat at a constant pressure of the base fluid, and $\kappa_{f}$ and $\kappa_{n}$ are the thermal conductivity of the base fluid and the solid fractions, respectively. The use of the abovementioned expression for $\left(\kappa_{\mathrm{nf}} / \kappa_{f}\right)$ is restricted to spherical nanoparticles only.

We establish the dimensionless variables as follows:

$$
\begin{aligned}
& x=\frac{x^{*}}{L}, \\
& y=\frac{y^{*}}{L}, \\
& t=t^{*} \omega, \\
& u=\frac{u^{*} \omega}{A}, \\
& p=\frac{p^{*}}{\rho A L}, \\
& \theta=\frac{T^{*}-T_{0}}{T_{1}-T_{0}} .
\end{aligned}
$$


By using the abovementioned dimensionless variables, we get

$$
\frac{(1+(1 / \beta))}{(1-\varphi)^{2.5}\left(1-\varphi+\left(\varphi \rho_{n} / \rho_{f}\right)\right)} \frac{\partial^{2} u}{\partial y^{2}}-\frac{\operatorname{Re}}{(1-\varphi)^{2.5}} \frac{\partial u}{\partial y}-\frac{1}{\left(1-\varphi+\left(\varphi \rho_{n} / \rho_{f}\right)\right)}\left(M^{2}+\frac{1}{(1-\varphi)^{2.5} \mathrm{Da}}\right) u-H^{2}\left(\frac{\partial u}{\partial t}+\frac{\partial p}{\partial x}\right)=0
$$

$$
\begin{aligned}
\frac{\left(\left(k_{\mathrm{nf}} / k_{f}\right)+(4 / 3) \mathrm{Rd}\right)}{\operatorname{Pr}} \frac{\partial^{2} \theta}{\partial y^{2}}-\frac{\operatorname{Re}\left(1-\varphi+\left(\varphi\left(\rho C_{p}\right)_{n} /\left(\rho C_{p}\right)_{f}\right)\right)}{(1-\varphi)^{2.5}} \frac{\partial \theta}{\partial y}+Q \theta= & -\frac{(1+(1 / \beta))}{(1-\varphi)^{2.5}} \operatorname{Ec}\left(\frac{\partial u}{\partial y}\right)^{2}-\operatorname{Ec}^{2} u^{2} \\
& +H^{2}\left(1-\varphi+\frac{\varphi\left(\rho C_{p}\right)_{n}}{\left(\rho C_{p}\right)_{f}} \frac{\partial \theta}{\partial t} .\right.
\end{aligned}
$$

The new boundary conditions are

$$
\begin{array}{ll}
u=0, \theta=0, & \text { at } y=0, \\
u=0, \theta=1, & \text { at } y=1,
\end{array}
$$

where $\mathrm{Da}=k / \Phi L^{2}$ is the Darcy number of the porous media, $H=L \sqrt{\omega} / \sqrt{\nu_{f}}$ is the frequency parameter, $M=B_{0} L \sqrt{\sigma} / \sqrt{\mu_{f}}$ is the Hartmann number, $\operatorname{Re}=V_{0} L / \nu_{f}$ is the cross-flow Reynolds number, $\mathrm{Ec}=((\mathrm{A})$ $\left.W)^{2} /\left(C_{p}\right)_{f}\left(T_{1}-T_{0}\right)\right)$ is the Eckert number, $Q=Q_{0} L^{2} /$ $\left(\left(\rho C_{p}\right)_{f} v_{f}\right)$ is the heat source/sink parameter, $\mathrm{Rd}=\left(4 T_{0}^{3} \sigma^{*} / k_{f} K^{*}\right)$ is the radiation parameter, and $\mathrm{Pr}=$ $\mu_{f}\left(C_{p}\right)_{f} / k_{f}$ is the Prandtl number.

\section{The Proposed Solution Method}

In order to solve equations (7) and (8) with the boundary conditions (9), we apply the perturbation technique. The velocity $u$ and temperature $\theta$ can be assumed to have the form

$$
\begin{aligned}
& u=u_{1}(y)+\varepsilon u_{2}(y) e^{\mathrm{it}}+\varepsilon^{2} u_{3}(y) e^{2 \mathrm{it}}, \\
& \theta=\theta_{1}(y)+\varepsilon \theta_{2}(y) e^{\mathrm{it}}+\varepsilon^{2} \theta_{3}(y) e^{2 \mathrm{it}} .
\end{aligned}
$$

Now, we substitute equations (10) and (11) into the equations (7) and (8); then, equating the coefficients of various powers of $\varepsilon$, we get

$$
\begin{gathered}
A_{1} u_{1}^{l l}-\frac{\operatorname{Re}}{(1-\varphi)^{2.5}} u_{1}^{l}-A_{2} u_{1}=-H^{2} \\
A_{1} u_{2}^{l l}-\frac{\operatorname{Re}}{(1-\varphi)^{2.5}} u_{2}^{l}-A_{6} u_{2}=-H^{2} \\
A_{1} u_{3}^{l l}-\frac{\operatorname{Re}}{(1-\varphi)^{2.5}} u_{3}^{l}-A_{10} u_{3}=-H^{2} \\
B_{1} \theta_{1}^{l l}-B_{2} \theta_{1}^{l}-Q \theta_{1}=-\frac{\operatorname{Ec}(1+(1 / \beta))}{(1-\varphi)^{2.5}} u_{1}^{l 2}-\mathrm{Ec}^{2} u_{1}^{2} \\
B_{1} \theta_{2}^{l l}-B_{2} \theta_{2}^{l}-B_{13} \theta_{2}=-\frac{2 \mathrm{Ec}(1+(1 / \beta))}{(1-\varphi)^{2.5}} u_{1}^{l} u_{2}^{l}-2 \mathrm{Ec}^{2} u_{1} u_{2},
\end{gathered}
$$

$$
B_{1} \theta_{3}^{l l}-B_{2} \theta_{3}^{l}-B_{25} \theta_{3}=-\frac{\operatorname{Ec}(1+(1 / \beta))}{(1-\varphi)^{2.5}} u_{2}^{l 2}-\operatorname{Ec} M^{2} u_{2}^{2}
$$

The new boundary conditions are

$$
\begin{array}{ll}
u_{1}=0, u_{2}=0, u_{3}=0, \theta_{1}=0, \theta_{2}=0, \theta_{3}=0, & \text { at } y=0, \\
u_{1}=0, u_{2}=0, u_{3}=0, \theta_{1}=1, \theta_{2}=0, \theta_{3}=0, & \text { at } y=1 .
\end{array}
$$

Solving the six ODEs (12)-(17) using the boundary conditions (18), we obtain 


$$
\begin{aligned}
u_{1}= & A_{3} e^{m_{1} y}+A_{4} e^{m_{2} y}+A_{5} \\
u_{2}= & A_{8} e^{m_{3} y}+A_{7} e^{m_{4} y}+A_{9} \\
u_{3}= & A_{11} e^{m_{5} y}+A_{12} e^{m_{6} y}+A_{13}, \\
\theta_{1}= & B_{12} e^{m_{7} y}+B_{11} e^{m_{8} y}+B_{5} e^{2 m_{1} y}+B_{6} e^{2 m_{2} y}+B_{7} e^{m_{1} y}+B_{8} e^{m_{2} y}+B_{9} e^{\left(m_{1}+m_{2}\right) y}+B_{10}, \\
\theta_{2}= & B_{23} e^{m_{9} y}+B_{24} e^{m_{10} y}+B_{14} e^{\left(m_{1}+m_{3}\right) y}+B_{15} e^{\left(m_{1}+m_{4}\right) y}+B_{16} e^{\left(m_{2}+m_{3}\right) y} \\
& +B_{17} e^{\left(m_{2}+m_{4}\right) y}+B_{18} e^{m_{1} y}+B_{19} e^{m_{2} y}+B_{20} e^{m_{3} y}+B_{21} e^{m_{4} y}+B_{22}, \\
\theta_{3}= & B_{43} e^{m_{11} y}+B_{42} e^{m_{12} y}+B_{26} e^{2 m_{3} y}+B_{27} e^{2 m_{4} y}+B_{28} e^{\left(m_{3}+m_{4}\right) y}+B_{29} e^{\left(m_{1}+m_{3}\right) y} \\
& +B_{30} e^{\left(m_{1}+m_{4}\right) y}+B_{31} e^{\left(m_{2}+m_{3}\right) y}+B_{32} e^{\left(m_{2}+m_{4}\right) y}+B_{33} e^{\left(m_{1}+m_{5}\right) y}+B_{34} e^{\left(m_{1}+m_{6}\right) y} \\
& +B_{35} e^{\left(m_{2}+m_{5}\right) y}+B_{36} e^{\left(m_{2}+m_{6}\right) y}+B_{37} e^{m_{3} y}+B_{38} e^{m_{4} y}+B_{39} e^{m_{5} y}+B_{40} e^{m_{6} y}+B_{41},
\end{aligned}
$$

where $m$ 's, $A s$, and $B s$ are the constants specified in Appendix. by

The dimensionless Nusselt number at the walls is given

$$
\mathrm{Nu}=-\left(\frac{\partial \theta}{\partial y}\right)_{y=0,1}=-\left(\theta_{1}^{\prime}(y)+\varepsilon \theta_{2}^{\prime}(y) e^{\mathrm{it}}+\varepsilon^{2} \theta_{3}^{\prime}(y) e^{2 \mathrm{it}}\right)_{y=0,1} .
$$

\section{Results and Discussion}

This segment presents the Casson nanofluid pulsating flow filling through a porous channel in the appearance of thermal radiation impacts. The governing physical systems are solved with the aid of the perturbation technique. MATLAB software assists in computing the system of equations through the perturbation method. Figures 2(a)$2(\mathrm{e})$ presents the effect of the frequency parameter $(H)$, Casson fluid parameter $(\beta)$,nanoparticle volume fraction $(\varphi)$, Hartmann number $(M)$, and Darcy number (Da) on the velocity distribution $u(y)$. Throughout the computation, the parameters are taken as $t=(\pi / 4), \operatorname{Re}=1, \phi=0.1$, $M=0.5, \beta=2, \mathrm{Da}=0.1, \varepsilon=0.01, \mathrm{Ec}=0.1, Q=0.5, \mathrm{Rd}=2$, $\operatorname{Pr}=21$, and $H=3$ unless otherwise stated.

Figures 2(a) and 2(b) exhibit the effect of $H$ and $\beta$ on velocity distribution $u(y)$, and it is noticed that $u(y)$ improves with an escalation in the frequency parameter $(H)$; furthermore, it is noted that the velocity is the increasing function of the Casson fluid parameter $(\beta)$ due to the diminishing behaviour of yield stress. Figure 2(c) illustrates the effect of $\varphi$ on $u(y)$. From this plot, we observed that the velocity upsurges with an escalation in the nanoparticle volume fraction $(\varphi)$. Figure $2(\mathrm{~d})$ exhibits the effect of $M$ on velocity profile $u(y)$. Here, as $M$ increases, reduction in velocity occurs because the retarding forces generated by the applied magnetic field act as resistive drag forces in the opposite direction to the flow. The influence of various values of the Darcy number (Da) on the velocity distribution $u(y)$ is presented in Figure 2(e). It is obtained from this graph that the velocity is the enhancing function of $\mathrm{Da}$. This is due to the Darcian drag force; it is known as the linear porous drag force and is inversely proportional to $\mathrm{Da}$ (see the third term in equation (7), i.e., $(-u / \mathrm{Da}))$. The Darcian drag force will enhance due to the rise in the absorbency of porous areas. Hence, there is an upsurge in velocity with higher range of Da.

Figures 3(a) and 3(b) display the influence of $(\varphi)$ and (Re) on the temperature distribution $\theta(y)$. The observations conclude that the temperature distribution $\theta(y)$ reduces with an upsurge in the nanoparticle volume fraction $(\varphi)$ and crossflow Reynolds number (Re). Figures 4(a) and 4(b) demonstrate the influence of the Prandtl number (Pr) and radiation parameter $(\mathrm{Rd})$ on the $\theta(y)$. Figure 4(a) depicts that the temperature distribution decreases with an increase in the Prandtl number (Pr). From Figure 4(b), one can observe a rise in $\theta(y)$ with intensification in $\mathrm{Rd}$.

The effect of the radiation parameter $(\mathrm{Rd})$,nanoparticle volume fraction $(\varphi)$, and Hartmann number $(M)$ on unsteady and steady temperature $\theta(y)$ is shown in Figures 5-7. Figure 5(a) shows that the unsteady temperature is oscillating with a decrease in the radiation parameter $(\mathrm{Rd})$ and attaining maximum near the walls. On the other hand, Figure 5(b) shows that the increase in the radiation parameter produces a diminishing tendency in steady temperature.

Figure 6(a) depicts the unsteady temperature growths with an increasing nanoparticle-volume fraction $(\varphi)$. From Figure 6(b), it is observed that the steady temperature increases towards the upper wall with a given increase in the nanoparticle-volume fraction $(\varphi)$, while the decrementing behaviour is noted in the lower wall. From Figure 7(a), we can observe that the Hartmann number $(M)$ enhancement generated the oscillating effect in the unsteady temperature. Figure 7(b) displays the lower temperature in the lower wall, while the higher temperature is noted in the upper wall.

Figure 8(a) shows the influence of nanoparticle-volume fraction $(\varphi)$ on the Nusselt number $(\mathrm{Nu})$ distribution. It is observed that the given increase in nanoparticle-volume fraction $(\varphi)$ results in the decrease of the Nusselt number 

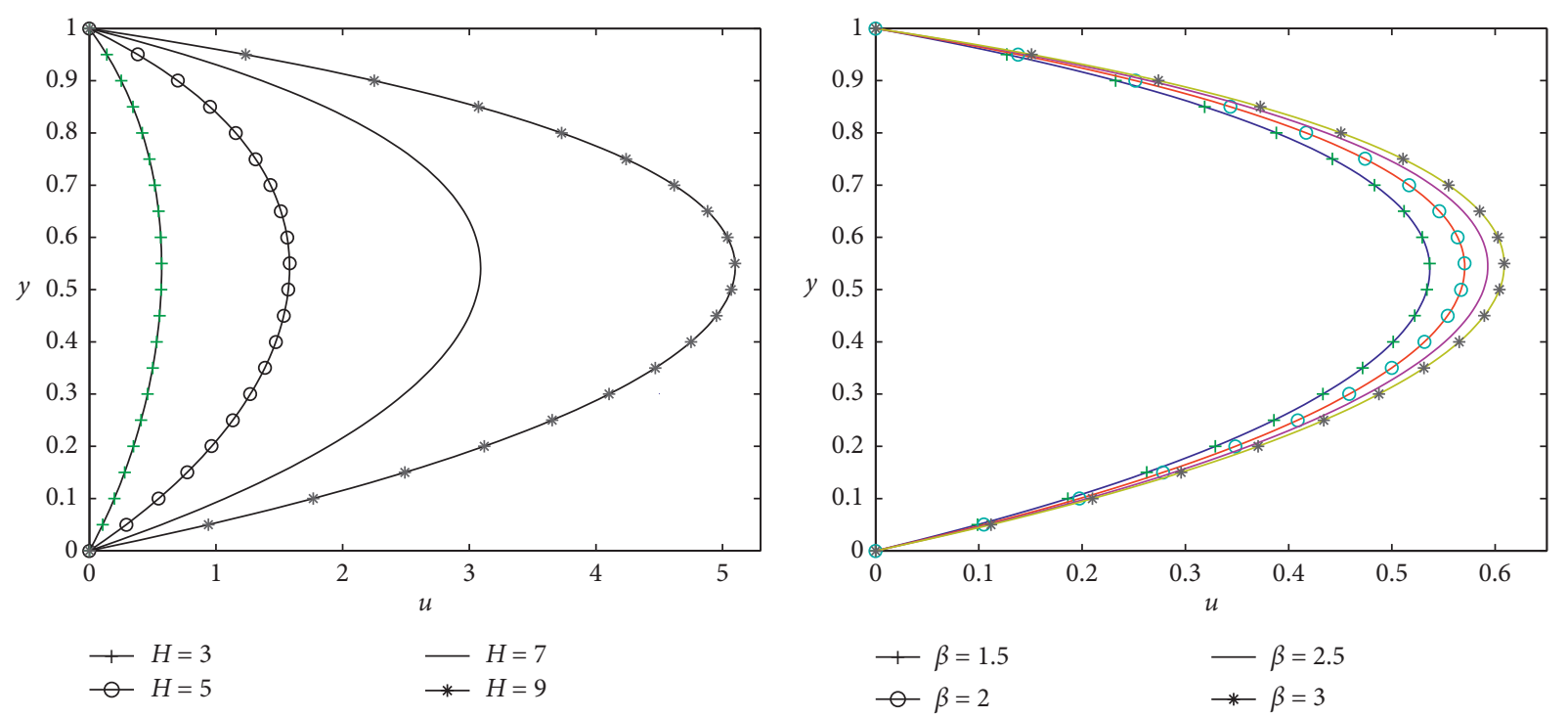

(a)

(b)
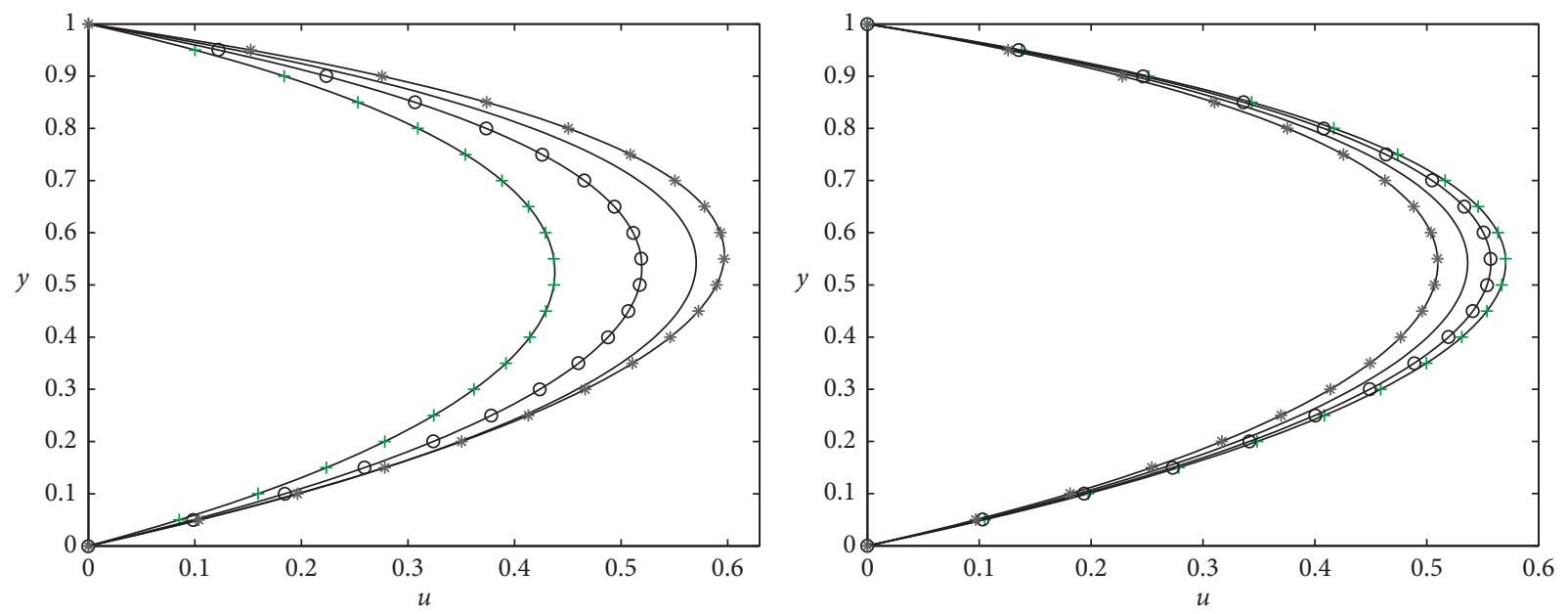

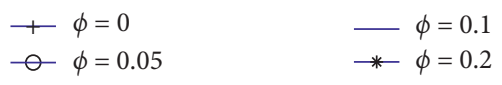

$+M=0.5$

$M=1.5$

(c)

(d)

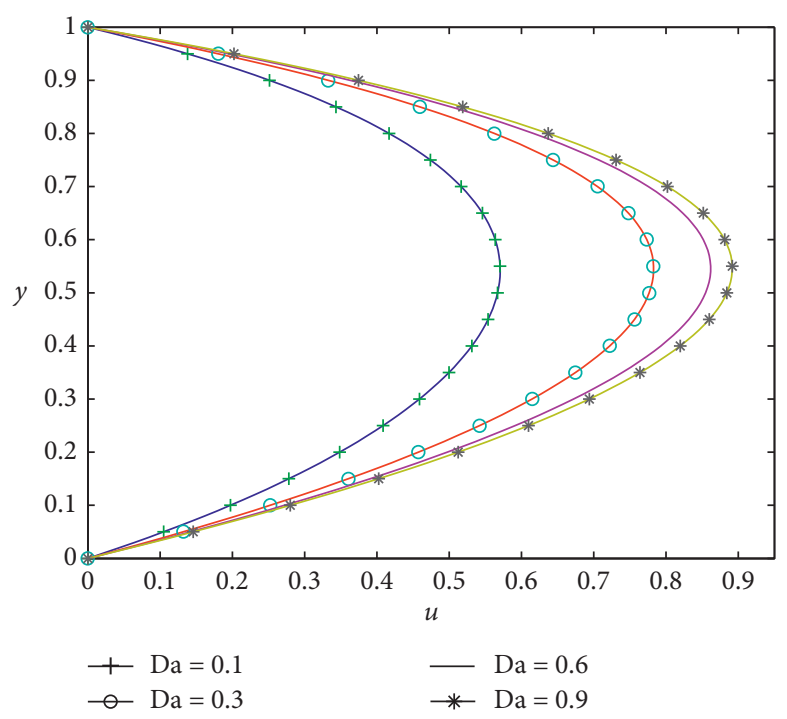

(e)

FIgURe 2: Velocity distribution for $t=\pi / 4, \varepsilon=0.01$, and $\operatorname{Re}=1$. (a) Effect of $H$ when $\phi=0.1, M=0.5, \mathrm{Da}=0.1$, and $\beta=2$. (b) Effect of $\beta$ when $\phi=0.1, M=0.5, \mathrm{Da}=0.1$, and $H=3$. (c) Effect of $\varphi$ when $M=0.5, \mathrm{Da}=0.1, \beta=2$, and $H=3$. (d) Effect of $M$ when $\phi=0.1, \mathrm{Da}=0.1, H=3$, and $\beta=2$. (e) Effect of $\mathrm{Da}$ when $\phi=0.1, M=0.5, H=3$, and $\beta=2$. 


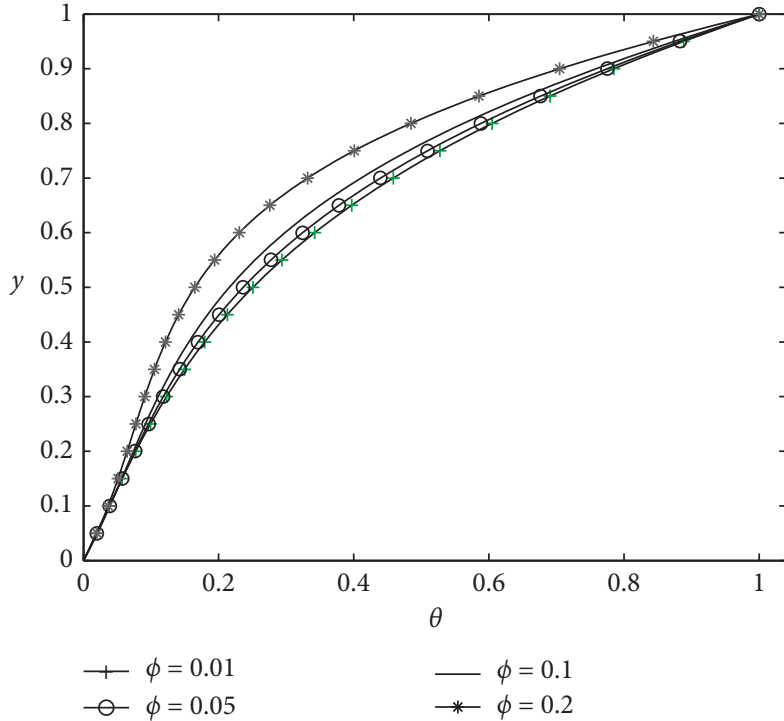

(a)

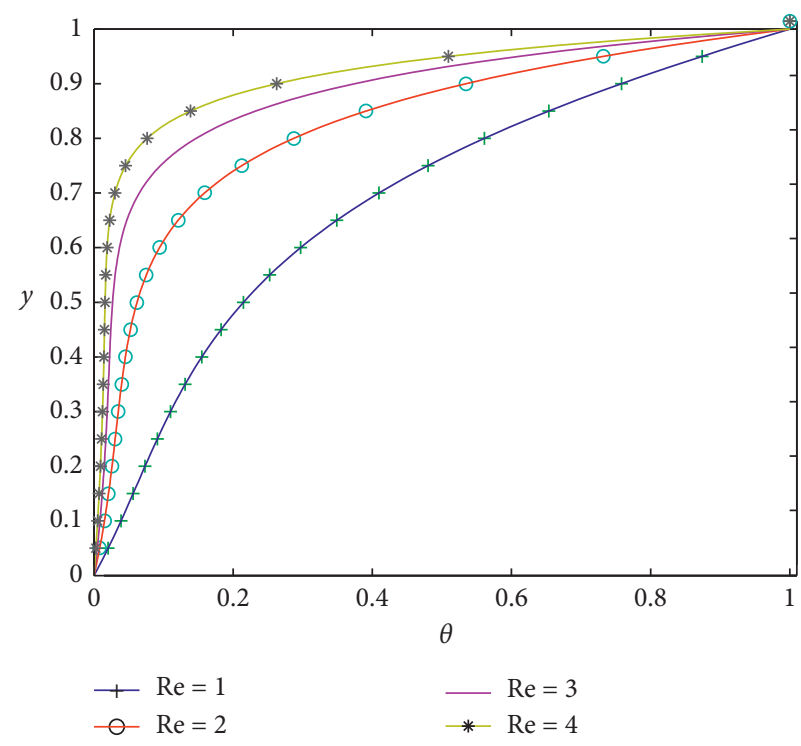

(b)

FiguRE 3: Temperature distribution for $\varepsilon=0.01, H=3, \beta=2, \mathrm{Ec}=0.1, \mathrm{Da}=0.1, \mathrm{Q}=0.5, \mathrm{Rd}=2, M=0.5, \operatorname{Pr}=21$, and $t=\pi / 4$. (a) Effect of $\phi$ when $\operatorname{Re}=1$. (b) Effect of $\operatorname{Re}$ when $\phi=0.1$.

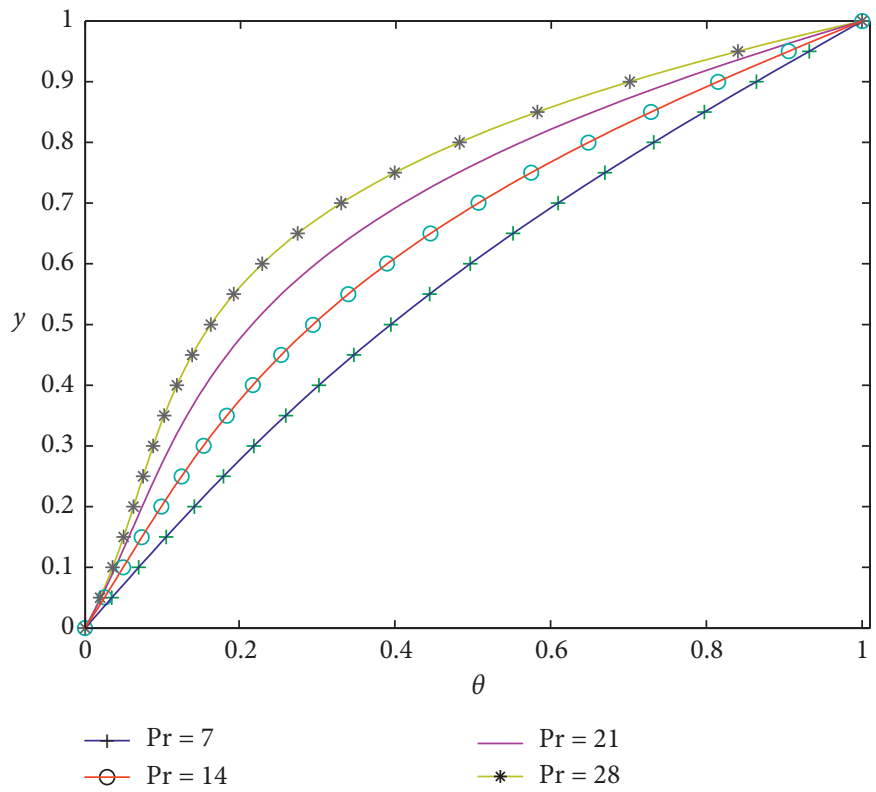

(a)

Figure 4: Continued. 


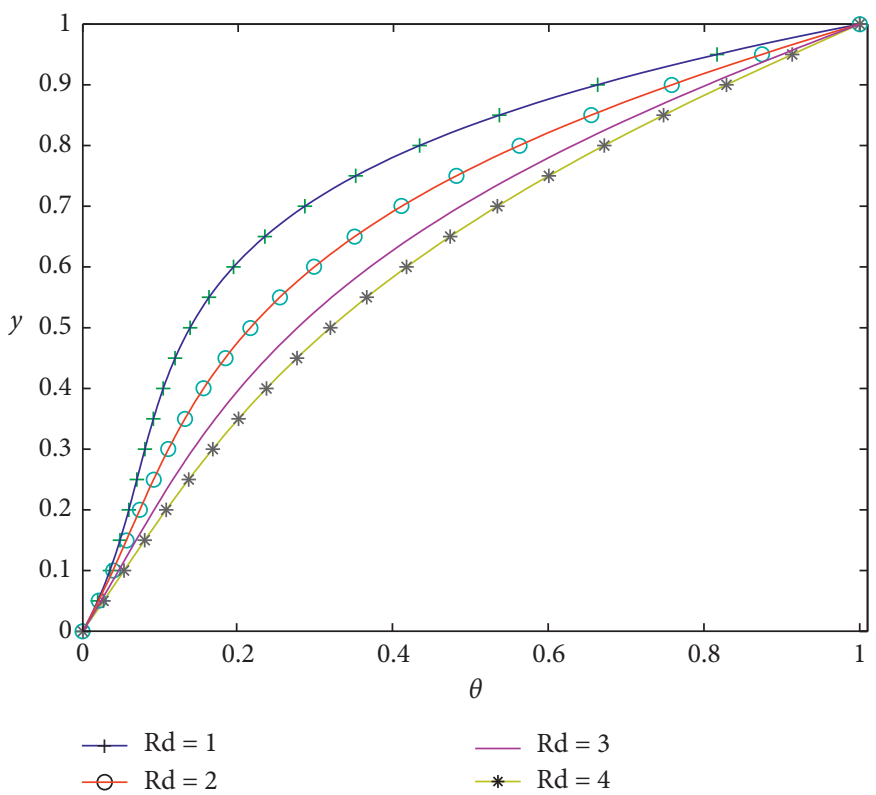

(b)

Figure 4: Temperature distribution for $t=\pi / 4, \varepsilon=0.01, H=3, \beta=2, \mathrm{Ec}=0.1, \mathrm{Q}=0.5, \mathrm{Da}=0.1, M=0.5, \operatorname{Re}=1$, and $\phi=0.1$. (a) Effect of $\operatorname{Pr}$ when $\mathrm{Rd}=2$, and (b) effect of $\mathrm{Rd}$ when $\operatorname{Pr}=21$.

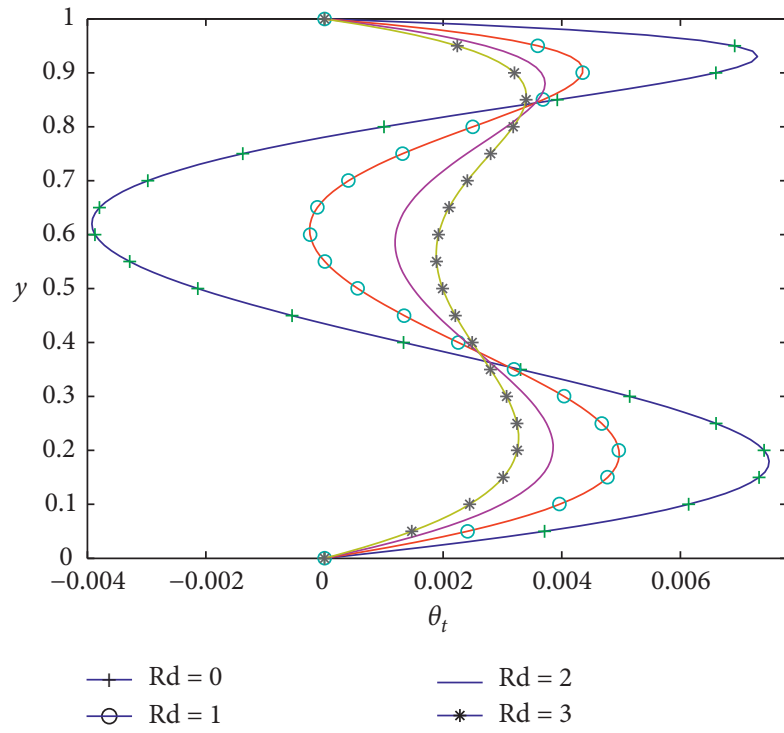

(a)

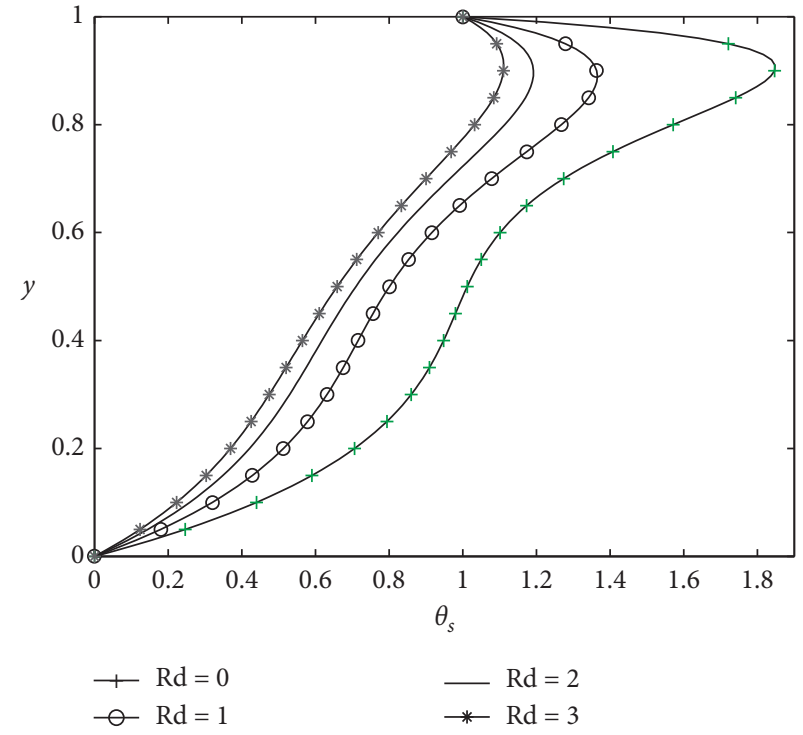

(b)

Figure 5: Effect of $\mathrm{Rd}$ on (a) unsteady and (b) steady temperature distributions when $t=\pi / 4, \varepsilon=0.01, H=3, \beta=2, \mathrm{Ec}=1, \mathrm{Q}$ $=0.5, \mathrm{Da}=0.1, M=0.5, \operatorname{Re}=1, \operatorname{Pr}=21$, and $\phi=0.1$.

$(\mathrm{Nu})$ at the lower wall, while it increases at the upper wall. Figure 8(b) shows the influence of the Hartmann number $(M)$ on the Nusselt number $(\mathrm{Nu})$ distribution. From this figure, we conclude that $\mathrm{Nu}$ rises at the lower wall and reduces at the upper wall for increasing the values of $M$.

Figure 9(a) shows the influence of the heat source/sink parameter $(Q)$ on the Nusselt number $(\mathrm{Nu})$ distribution. It is observed that the given increase in heat source at the lower wall results in the decrease of the Nusselt number $(\mathrm{Nu})$. In contrast, it increases at the upper wall. The increase in heat sink at the lower wall increases the Nusselt number $(\mathrm{Nu})$, while it decreases at the upper wall. Figure 9(b) shows the influence of radiation ( $\mathrm{Rd}$ ) parameter on the Nusselt number $(\mathrm{Nu})$ distribution. It is observed that the given 


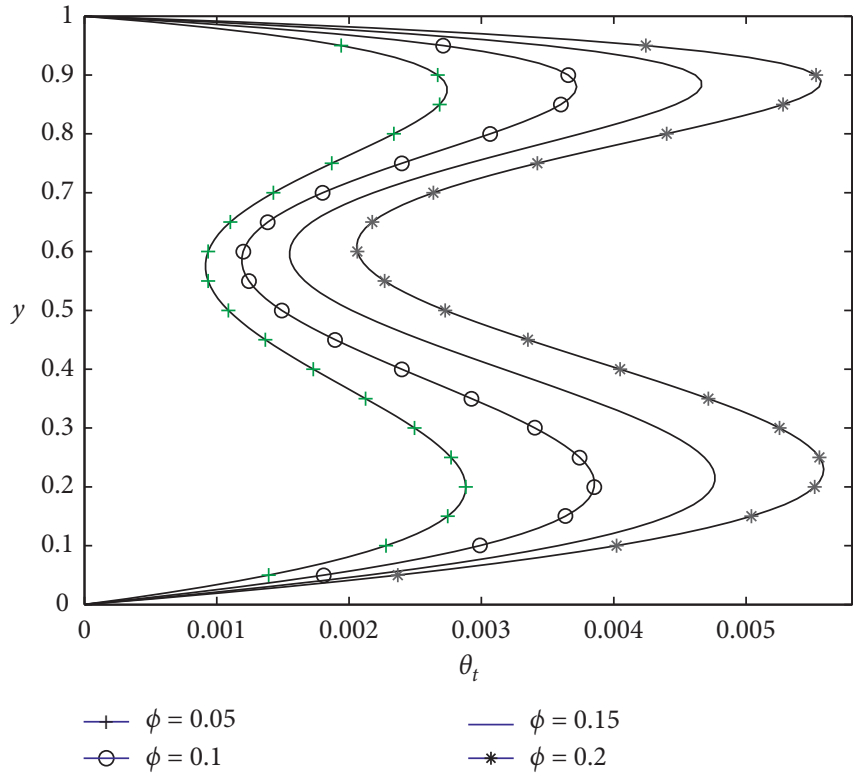

(a)
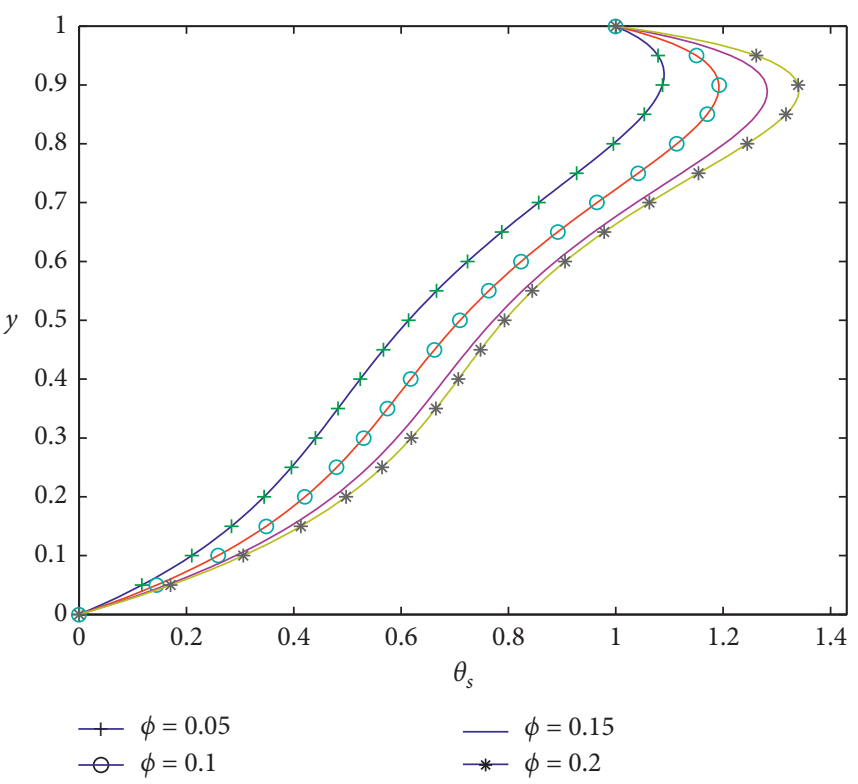

(b)

Figure 6: Effect of $\phi$ on (a) unsteady and (b) steady temperature distributions when $t=\pi / 4, \varepsilon=0.01, H=3, \beta=2, \mathrm{Ec}=1, \mathrm{Q}=0.5, \mathrm{Da}=$ $0.1, M=0.5, \operatorname{Re}=1, \operatorname{Pr}=21$, and $\mathrm{Rd}=2$.

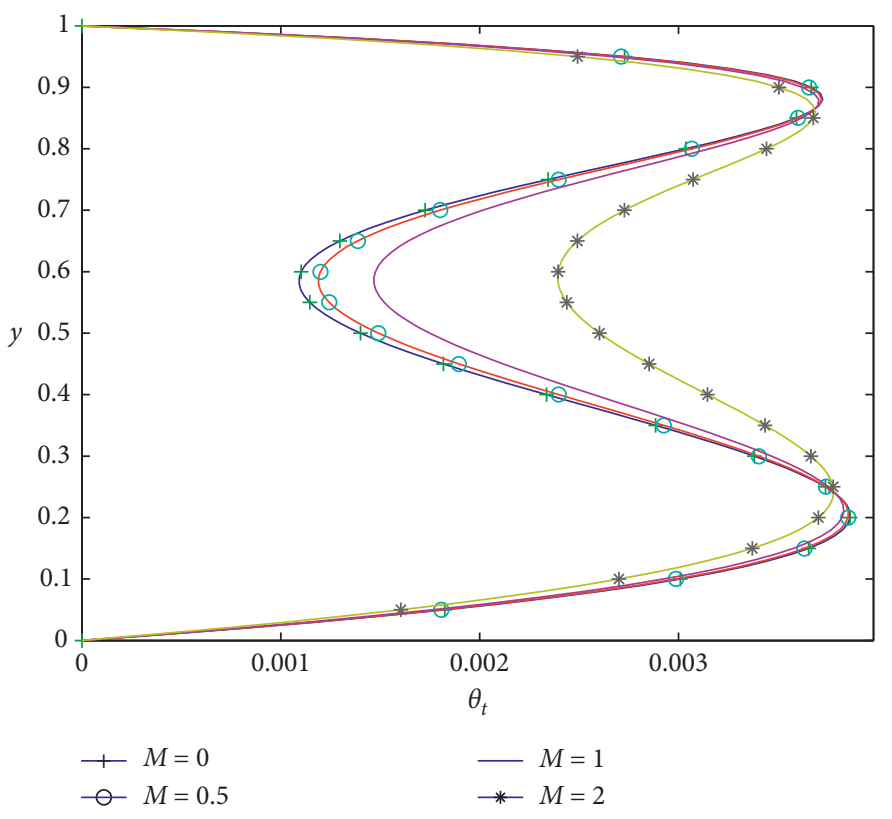

(a)

Figure 7: Continued. 


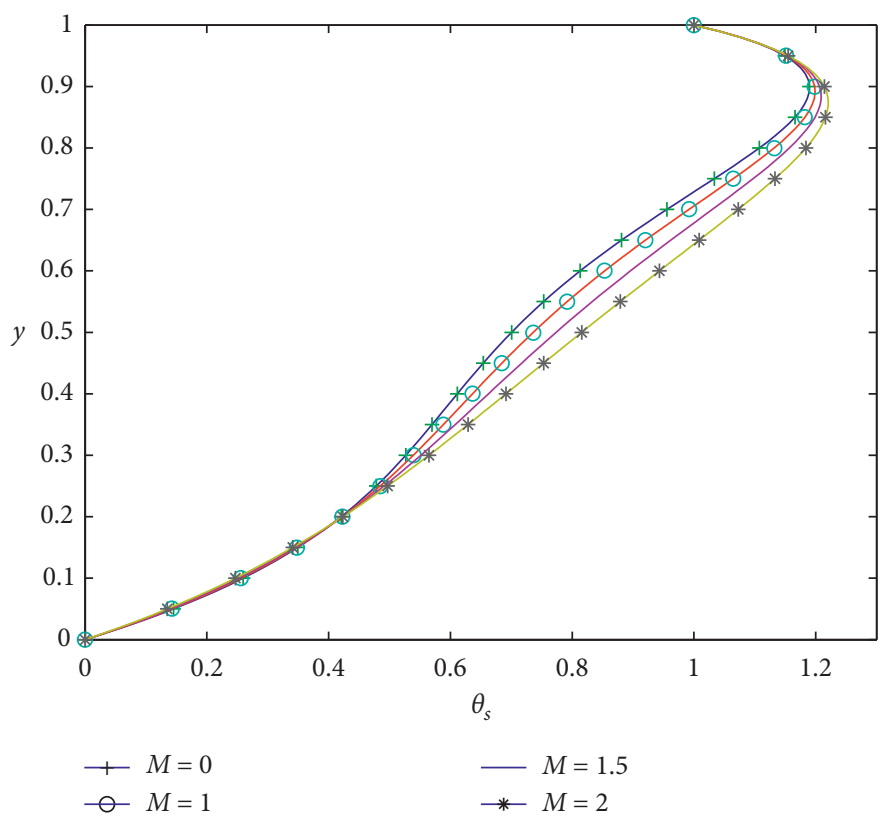

(b)

Figure 7: Effect of $M$ on (a) unsteady and (b) steady temperature distributions when $t=\pi / 4, \varepsilon=0.01, H=3, \beta=2, \mathrm{Ec}=1, \mathrm{Q}=0.5, \mathrm{Da}=$ $0.1, \varphi=0.1, \operatorname{Re}=1, \operatorname{Pr}=21$, and $\mathrm{Rd}=2$.

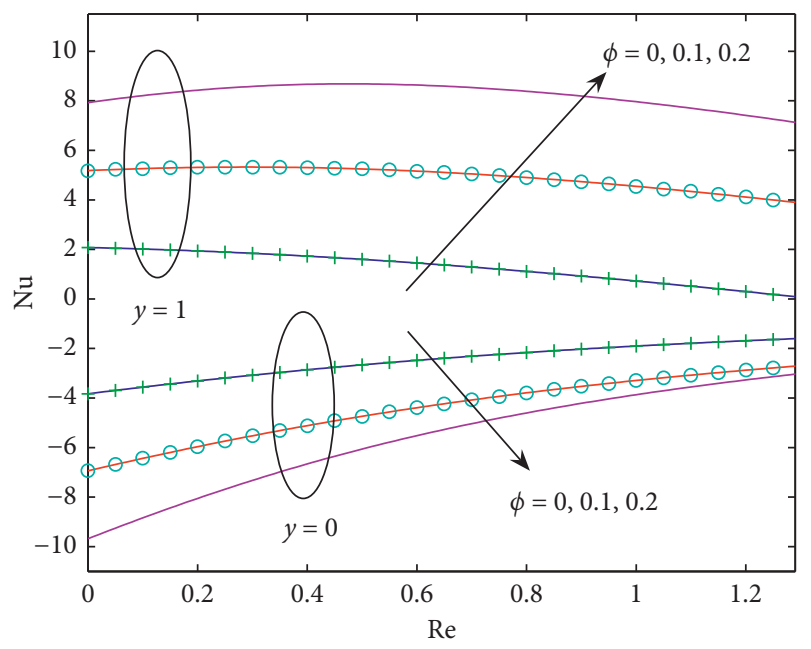

(a)

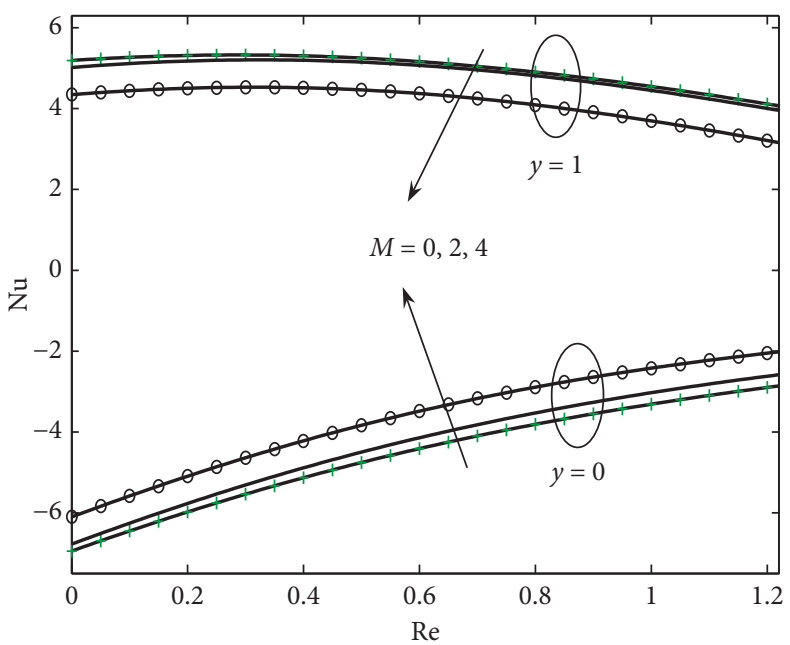

(b)

FIGURE 8: Nusselt number distribution for $t=\pi / 4, \varepsilon=0.01, H=3, \beta=2, \mathrm{Ec}=1, \mathrm{Da}=0.1, \mathrm{Rd}=2, \operatorname{Re}=1$, and $\operatorname{Pr}=21, \mathrm{Q}=0.5$. (a) Effect of $\phi$ when $M=0.5$, and (b) effect of $M$ when $\phi=0.1$.

increase in radiation parameter $(\mathrm{Rd})$ results in the decrease of the Nusselt number $(\mathrm{Nu})$ at the upper wall, while it increases at the lower wall.

Table 1 shows the comparison of results of Nusselt number $\mathrm{Nu}$ with Srinivas et al. [18]. The matching of these results is made with the heat transfer rate of Newtonian and non-Newtonian. From this result, we can conclude that the present computation is an efficient one.
Table 2 presents the variations of the Nusselt number $(\mathrm{Nu})$. The nanofluid is taken as the nanoparticle volume fraction $\varphi=0.1$. From this table, $\mathrm{Nu}$ enhanced at the lower wall for the higher values of $M, \mathrm{Re}$, and $\mathrm{Rd}$, while the inverse trend is noticed for an enormous $Q$ value. However, this behaviour is reversed at the upper wall. It is also observed that, with increasing nanoparticle volume fraction $(\varphi), \mathrm{Nu}$ decreases at the lower wall, while it is increasing at the upper wall. 


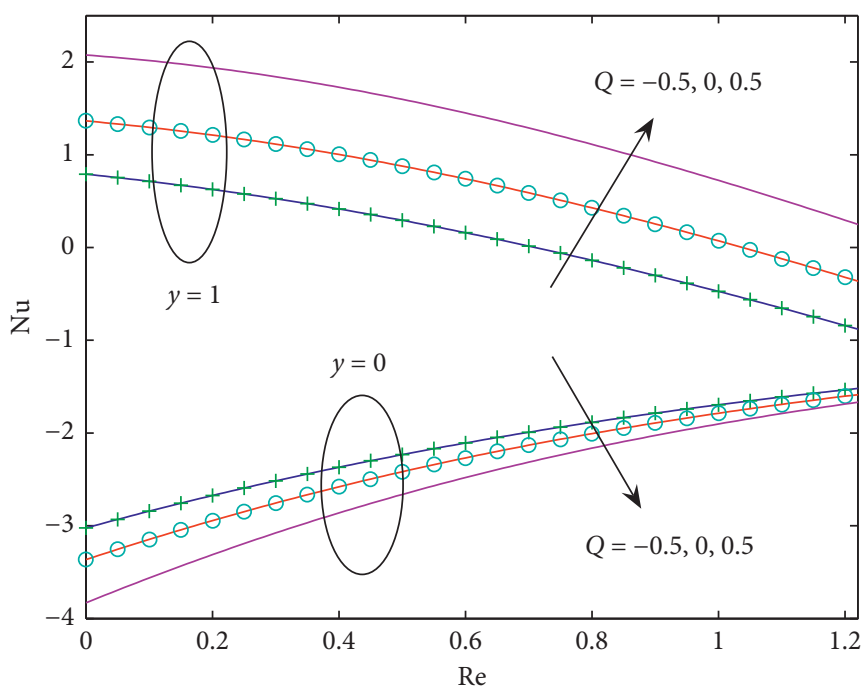

(a)

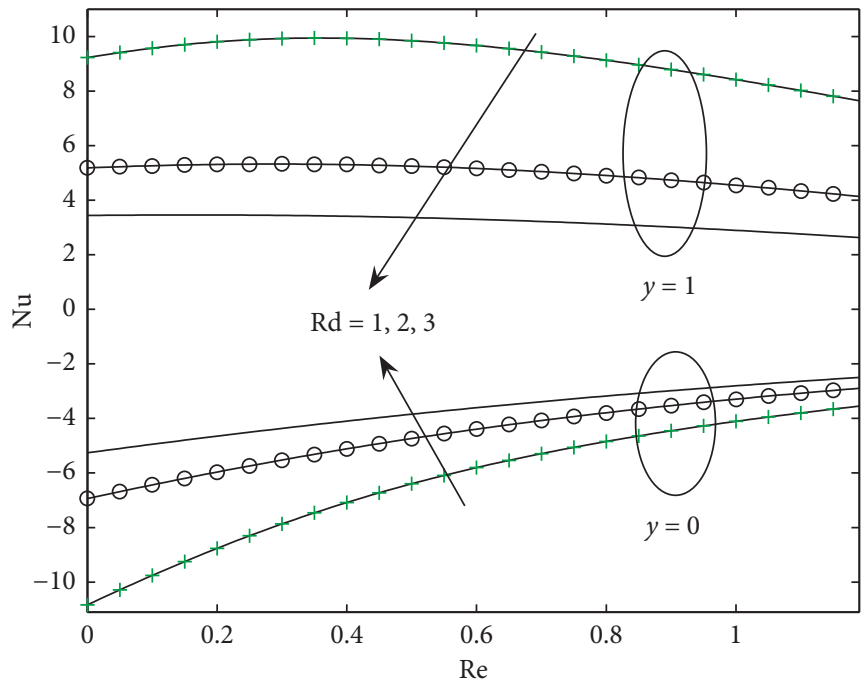

(b)

Figure 9: Nusselt number distribution for $t=\pi / 4, \varepsilon=0.01, H=3, \beta=2$, Ec $=1, \mathrm{Da}=0.1, M=0.5, \operatorname{Re}=1$, and $\operatorname{Pr}=21$. (a) Effect of $Q$ when $\mathrm{Rd}=2$ and $\phi=0$. (b) Effect of $\mathrm{Rd}$ when $Q=0.5$ and $\phi=0.1$.

Table 1: Comparison of the Nusselt number for Newtonian and non-Newtonian fluids with the work of Srinivas et al. [18] for the limiting case of $\phi=0, \varepsilon=0.01, H=3, \beta=2, \mathrm{Ec}=1, \mathrm{Da}=0.1, \operatorname{Pr}=21$, and $t=\pi / 4$.

\begin{tabular}{|c|c|c|c|c|c|c|c|c|c|}
\hline \multirow[b]{2}{*}{ Parameter } & \multirow[b]{2}{*}{ Values } & \multicolumn{4}{|c|}{$\mathrm{Nu}=-(\partial \theta / \partial y)_{y=0}$} & \multicolumn{4}{|c|}{$\mathrm{Nu}=-(\partial \theta / \partial y)_{y=1}$} \\
\hline & & $\begin{array}{c}\text { Newtonian } \\
{[18]}\end{array}$ & $\begin{array}{c}\text { Newtonian } \\
\text { (present) }\end{array}$ & $\begin{array}{c}\text { Non- } \\
\text { Newtonian } \\
{[18]}\end{array}$ & $\begin{array}{c}\text { Non- } \\
\text { Newtonian } \\
\text { (present) }\end{array}$ & $\begin{array}{c}\text { Newtonian } \\
{[18]}\end{array}$ & $\begin{array}{c}\text { Newtonian } \\
\text { (present) }\end{array}$ & $\begin{array}{c}\text { Non- } \\
\text { Newtonian } \\
{[18]}\end{array}$ & $\begin{array}{c}\text { Non- } \\
\text { Newtonian } \\
\text { (present) }\end{array}$ \\
\hline \multirow{3}{*}{ M } & 0 & -2.7033 & -2.7033 & -2.6361 & -2.6361 & 3.1771 & 3.1771 & 2.7457 & 2.7457 \\
\hline & 2 & -2.2726 & -2.2726 & -2.2307 & -2.2307 & 3.5754 & 3.5754 & 2.7261 & 2.7261 \\
\hline & 4 & -1.5615 & -1.5615 & -1.5380 & -1.5380 & 2.6740 & 2.6740 & 1.8116 & 1.8116 \\
\hline \multirow{3}{*}{$\operatorname{Re}$} & 0 & -7.6829 & -7.6829 & -7.1678 & -7.1678 & 6.1846 & 6.1846 & 5.6695 & 5.6695 \\
\hline & 1 & -2.2726 & -2.2726 & -2.2307 & -2.2307 & 3.5754 & 3.5754 & 2.7261 & 2.7261 \\
\hline & 2 & -1.1585 & -1.1585 & -1.2067 & -1.2067 & -1.9577 & -1.9577 & -2.8056 & -2.8056 \\
\hline \multirow{3}{*}{ Q } & 0 & -2.5506 & -2.5506 & -2.4868 & -2.4868 & 1.6763 & 1.6763 & 1.2142 & 1.2142 \\
\hline & 0.5 & -2.6712 & -2.6712 & -2.6064 & -2.6064 & 3.2306 & 3.2306 & 2.7556 & 2.7556 \\
\hline & 1 & -2.8333 & -2.8333 & -2.7671 & -2.7671 & 5.4356 & 5.4356 & 4.9425 & 4.9425 \\
\hline \multirow{3}{*}{$\mathrm{Rd}$} & 0 & -4.2702 & -4.2702 & -4.1034 & -4.1034 & 19.2777 & 19.2777 & 17.4188 & 17.4188 \\
\hline & 1 & -3.3850 & -3.3850 & -3.2904 & -3.2904 & 6.2590 & 6.2590 & 5.4984 & 5.4984 \\
\hline & 2 & -2.8333 & -2.8333 & -2.7671 & -2.7671 & 3.2303 & 3.2303 & 2.7556 & 2.7556 \\
\hline
\end{tabular}




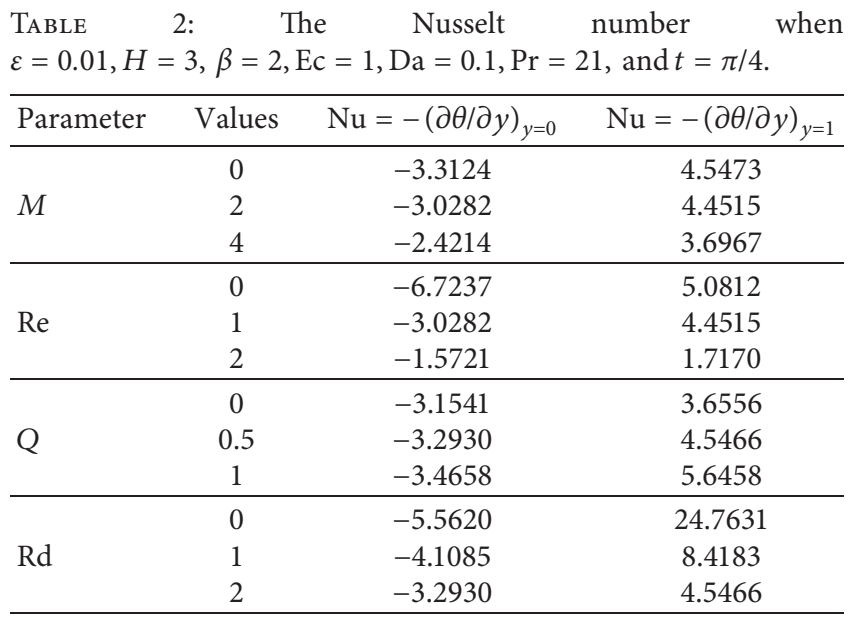

\section{Conclusions}

The present study deals with the Casson nanofluid flow through a porous channel with radiation and MHD effects. The pulsating flow is included in the flow problem. The analytical technique (perturbation) is utilized to compute the physical system. The considered problem is essential as the flow of nanofluids in the porous channel has enormous applications in industrial and biological research such as crude oil purification and blood flow in the heart and lungs. The necessary upshots of this research are noted as follows:

(1) The fluid velocity enhances for higher values of the frequency parameter, Casson fluid parameter, nanoparticle volume fraction, and Darcy number. On the other hand, the reverse impact is observed for larger values of the Hartmann number.

(2) Improved values of the cross Reynolds number and the radiation parameter produce a decrement in temperature.

(3) The Nusselt number declines at the lower wall and increases at the upper wall with increasing nanoparticle volume fraction.

(4) Diminishing behaviour of the Nusselt number is noted at the upper wall, and it is increasing at the lower wall with an augmentation in the Hartmann number, heat sink, and radiation parameters.

(5) The hydrodynamic case can be obtained for the present problem by taking $M=0$.

\section{Nomenclature}

A: $\quad$ A constant defined in equation (1)

$B_{0}$ : Uniform magnetic field

$C$ pnf: $\quad$ Specific heat at a constant pressure of nanofluid

Da: Darcy number of the porous media

Ec: $\quad$ Eckert number

$H$ : $\quad$ Frequency parameter

$k$ : $\quad$ Permeability of the porous media

$\kappa_{\text {nf: }} \quad$ Thermal conductivity of the nanofluid

M: $\quad$ Hartmann number

Nu: $\quad$ Nusselt number

Pr: $\quad$ Prandtl number

$p^{*}: \quad$ Pressure

Q: $\quad$ Heat source/ sink parameter

Re: Cross-flow Reynolds number

Rd: Radiation parameter

$T_{0}$ : The temperature at the lower wall

$T_{1}$ : The temperature at the upper wall

$\Phi: \quad$ The porosity of the porous medium

$\sigma: \quad$ Electrical conductivity of the fluid

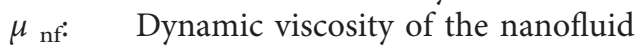

$\rho$ nf: $\quad$ The density of the nanofluid

$v_{\mathrm{nf}}$ : Kinematic viscosity of the nanofluid

$\varphi: \quad$ Nanoparticle volume fraction

$\left(\rho C_{p}\right)_{\mathrm{nf}}$ : Heat capacity of the nanofluid

$\omega: \quad$ Frequency

$f: \quad$ Fluid fraction

$n$ : Nanoparticle fraction

nf: Nanofluid.

\section{Appendix}

$$
\begin{aligned}
& A_{1}=\frac{((1 / \beta)+1)}{\left(1-\varphi+\left(\varphi \rho_{n} / \rho_{f}\right)\right)(1-\varphi)^{2.5}} \\
& A_{2}=\frac{1}{\left(1-\varphi+\left(\varphi \rho_{n} / \rho_{f}\right)\right)}\left(M^{2}+\frac{1}{(1-\varphi)^{2.5} \mathrm{Da}}\right) \text {; } \\
& A_{5}=\frac{H^{2}}{A_{2}} \\
& m_{1,2}=\frac{\left(\operatorname{Re} /(1-\varphi)^{2.5}\right) \pm \sqrt{\left(\operatorname{Re} /(1-\varphi)^{2.5}\right)^{2}+4 A_{1} A_{2}}}{2 A_{1}}, \\
& A_{3}=\frac{A_{5}\left(e^{m_{2}}-1\right)}{\left(e^{m_{1}}-e^{m_{2}}\right)} \\
& A_{4}=-\frac{A_{5}\left(e^{m_{1}}-1\right)}{\left(e^{m_{1}}-e^{m_{2}}\right)} \\
& A_{6}=\frac{1}{\left(1-\varphi+\left(\varphi \rho_{n} / \rho_{f}\right)\right)}\left(M^{2}+\frac{1}{(1-\varphi)^{2.5} \mathrm{Da}}\right)+i H^{2} \text {; } \\
& A_{9}=\frac{H^{2}}{A_{6}} \\
& m_{3,4}=\frac{\left(\operatorname{Re} /(1-\varphi)^{2.5}\right) \pm \sqrt{\left(\operatorname{Re} /(1-\varphi)^{2.5}\right)^{2}+4 A_{1} A_{6}}}{2 A_{1}}, \\
& A_{7}=\frac{A_{9}\left(e^{m_{3}}-1\right)}{\left(e^{m_{4}}-e^{m_{3}}\right)}
\end{aligned}
$$




$$
\begin{aligned}
& A_{8}=\frac{A_{9}\left(e^{m_{4}}-1\right)}{\left(e^{m_{3}}-e^{m_{4}}\right)} \\
& A_{10}=\frac{1}{\left(1-\varphi+\left(\varphi \rho_{n} / \rho_{f}\right)\right)}\left(M^{2}+\frac{1}{(1-\varphi)^{2.5} \mathrm{Da}}\right)+2 i H^{2} \\
& A_{13}=\frac{H^{2}}{A_{10}} \text {, } \\
& m_{5,6}=\frac{\left(\operatorname{Re} /(1-\varphi)^{2.5}\right) \pm \sqrt{\left(\operatorname{Re} /(1-\varphi)^{2.5}\right)^{2}+4 A_{1} A_{10}}}{2 A_{1}}, \\
& A_{11}=\frac{A_{13}\left(e^{m_{6}}-1\right)}{\left(e^{m_{5}}-e^{m_{6}}\right)} \\
& A_{12}=\frac{A_{13}\left(e^{m_{5}}-1\right)}{\left(e^{m_{6}}-e^{m_{5}}\right)} \\
& B_{1}=\frac{\left(\left(k_{\mathrm{nf}} / k_{f}\right)+(4 / 3) \mathrm{Rd}\right)}{\operatorname{Pr}}, \\
& B_{2}=\frac{\operatorname{Re}\left(1-\varphi+\left(\varphi\left(\rho C_{p}\right)_{n} /\left(\rho C_{p}\right)_{f}\right)\right)}{(1-\varphi)^{2.5}}, \\
& m_{7,8}=\frac{B_{2} \pm \sqrt{B_{2}^{2}+4 B_{1} Q}}{2 B_{1}}, \\
& B_{3}=\frac{(1+(1 / \beta))}{(1-\varphi)^{2.5}} \mathrm{Ec}, \\
& B_{4}=M E c \text {, } \\
& B_{5}=-\frac{B_{3} m_{1}^{2} A_{3}^{2}+B_{4} A_{3}^{2}}{4 m_{1}^{2} B_{1}-2 m_{1} B_{2}+Q}, \\
& B_{6}=-\frac{B_{3} m_{2}^{2} A_{4}^{2}+B_{4} A_{4}^{2}}{4 m_{2}^{2} B_{1}-2 m_{2} B_{2}+Q}, \\
& B_{7}=\frac{2 B_{4} A_{3} A_{5}}{m_{1}^{2} B_{1}-m_{1} B_{2}+Q} \text {, } \\
& B_{8}=\frac{2 B_{4} A_{4} A_{5}}{m_{2}^{2} B_{1}-m_{2} B_{2}+Q} \text {, } \\
& B_{9}=-\frac{2 B_{3} m_{1} m_{2} A_{3} A_{4}+2 B_{4} A_{3} A_{4}}{\left(m_{1}+m_{2}\right)^{2} B_{1}-\left(m_{1}+m_{2}\right) B_{2}+Q}, \\
& B_{10}=\frac{B_{4} A_{5}^{2}}{Q}, \\
& B_{11}=\frac{1}{\left(e^{m_{8}}-e^{m_{7}}\right)}\left(1+B_{5}\left(e^{m_{7}}-e^{2 m_{1}}\right)-B_{6}\left(e^{m_{7}}-e^{2 m_{2}}\right)\right. \\
& +B_{7}\left(e^{m_{7}}-e^{m_{1}}\right)+B_{8}\left(e^{m_{7}}-e^{m_{2}}\right) \\
& \left.+B_{9}\left(e^{m_{7}}-e^{\left(m_{1}+m_{2}\right)}\right)+B_{10}\left(e^{m_{7}}-1\right)\right), \\
& B_{12}=-\left(B_{11}+B_{10}+B_{9}+B_{8}+B_{7}+B_{6}+B_{5}\right) \text {, } \\
& B_{13}=Q-i\left(1-\varphi+\frac{\varphi\left(\rho C_{p}\right)_{n}}{\left(\rho C_{p}\right)_{f}}\right) H^{2} \text {, } \\
& m_{9,10}=\frac{B_{2} \pm \sqrt{B_{2}^{2}+4 B_{1} B_{13}}}{2 B_{1}},
\end{aligned}
$$

$$
B_{14}=-\frac{2 B_{3} m_{1} m_{3} A_{3} A_{8}+2 B_{4} A_{3} A_{8}}{\left(m_{1}+m_{3}\right)^{2} B_{1}-\left(m_{1}+m_{2}\right) B_{2}+B_{13}},
$$$$
B_{15}=-\frac{2 B_{3} m_{1} m_{4} A_{3} A_{7}+2 B_{4} A_{3} A_{7}}{\left(m_{1}+m_{4}\right)^{2} B_{1}-\left(m_{1}+m_{4}\right) B_{2}+B_{13}},
$$$$
B_{16}=-\frac{2 B_{3} m_{2} m_{3} A_{4} A_{8}+2 B_{4} A_{4} A_{8}}{\left(m_{2}+m_{3}\right)^{2} B_{1}-\left(m_{2}+m_{3}\right) B_{2}+B_{13}},
$$$$
B_{17}=-\frac{2 B_{3} m_{2} m_{4} A_{4} A_{7}+2 B_{4} A_{4} A_{7}}{\left(m_{2}+m_{4}\right)^{2} B_{1}-\left(m_{2}+m_{4}\right) B_{2}+B_{13}},
$$$$
B_{18}=\frac{2 B_{4} A_{3} A_{9}}{m_{1}^{2} B_{1}-m_{1} B_{2}+B_{13}} \text {, }
$$$$
B_{19}=\frac{2 B_{4} A_{4} A_{9}}{m_{2}^{2} B_{1}-m_{2} B_{2}+B_{13}} \text {, }
$$$$
B_{20}=\frac{2 B_{4} A_{5} A_{8}}{m_{3}^{2} B_{1}-m_{3} B_{2}+B_{13}} \text {, }
$$$$
B_{21}=\frac{2 B_{4} A_{5} A_{7}}{m_{4}^{2} B_{1}-m_{4} B_{2}+B_{13}} \text {, }
$$$$
B_{22}=-\frac{2 B_{4} A_{5} A_{9}}{B_{13}},
$$$$
B_{24}=\frac{1}{\left(e^{m_{10}}-e^{m_{9}}\right)}\left(B_{14}\left(e^{m_{9}}-e^{\left(m_{1}+m_{3}\right)}\right)\right.
$$$$
+B_{15}\left(e^{m_{9}}-e^{\left(m_{1}+m_{4}\right)}\right)-B_{16}\left(e^{m_{9}}-e^{\left(m_{2}+m_{3}\right)}\right)
$$$$
+B_{17}\left(e^{m_{9}}-e^{\left(m_{4}+m_{2}\right)}\right)+B_{18}\left(e^{m_{9}}-e^{m_{1}}\right)
$$$$
+B_{19}\left(e^{m_{9}}-e^{m_{2}}\right)+B_{20}\left(e^{m_{9}}-e^{m_{3}}\right)
$$$$
+B_{21}\left(e^{m_{9}}-e^{m_{4}}\right)+B_{22}\left(e^{m_{9}}-1\right) \text {, }
$$$$
B_{23}=-\left(B_{16}+B_{14}+B_{15}+B_{17}+B_{18}+B_{19}\right.
$$$$
\left.+B_{22}+B_{24}+B_{20}+B_{21}\right) \text {, }
$$$$
B_{25}=Q-2 i\left(1-\varphi+\frac{\varphi\left(\rho C_{p}\right)_{n}}{\left(\rho C_{p}\right)_{f}}\right) H^{2} \text {, }
$$$$
m_{11,12}=\frac{B_{2} \pm \sqrt{B_{2}^{2}+4 B_{1} B_{25}}}{2 B_{1}},
$$$$
B_{26}=-\frac{B_{3} m_{3}^{2} A_{8}^{2}+B_{4} A_{8}^{2}}{m_{3}^{2} B_{1}-2 m_{3} B_{2}+B_{25}},
$$$$
B_{27}=-\frac{B_{3} m_{4}^{2} A_{7}^{2}+B_{4} A_{7}^{2}}{m_{4}^{2} B_{1}-2 m_{4} B_{2}+B_{25}},
$$$$
B_{28}=-\frac{2 B_{3} m_{3} m_{4} A_{8} A_{7}+2 B_{4} A_{8} A_{7}}{\left(m_{3}+m_{4}\right)^{2} B_{1}-\left(m_{3}+m_{4}\right) B_{2}+B_{25}},
$$$$
B_{29}=-\frac{2 B_{3} m_{1} m_{3} A_{3} A_{8}}{\left(m_{1}+m_{3}\right)^{2} B_{1}-\left(m_{1}+m_{3}\right) B_{2}+B_{25}} \text {, }
$$$$
B_{30}=-\frac{2 B_{3} m_{1} m_{4} A_{3} A_{7}}{\left(m_{1}+m_{4}\right)^{2} B_{1}-\left(m_{1}+m_{4}\right) B_{2}+B_{25}} \text {, }
$$$$
B_{31}=-\frac{2 B_{3} m_{2} m_{3} A_{4} A_{8}}{\left(m_{2}+m_{3}\right)^{2} B_{1}-\left(m_{2}+m_{3}\right) B_{2}+B_{25}},
$$ 


$$
\begin{aligned}
& B_{32}=-\frac{2 B_{3} m_{2} m_{4} A_{4} A_{7}}{\left(m_{2}+m_{4}\right)^{2} B_{1}-\left(m_{2}+m_{4}\right) B_{2}+B_{25}}, \\
& B_{33}=-\frac{2 B_{4} A_{3} A_{11}}{\left(m_{1}+m_{5}\right)^{2} B_{1}-\left(m_{1}+m_{5}\right) B_{2}+B_{25}} \text {, } \\
& B_{34}=-\frac{2 B_{4} A_{3} A_{12}}{\left(m_{1}+m_{6}\right)^{2} B_{1}-\left(m_{1}+m_{6}\right) B_{2}+B_{25}} \text {, } \\
& B_{35}=-\frac{2 B_{4} A_{4} A_{11}}{\left(m_{2}+m_{5}\right)^{2} B_{1}-\left(m_{2}+m_{5}\right) B_{2}+B_{25}} \text {, } \\
& B_{36}=-\frac{2 B_{4} A_{4} A_{12}}{\left(m_{2}+m_{6}\right)^{2} B_{1}-\left(m_{2}+m_{6}\right) B_{2}+B_{25}} \text {, } \\
& B_{37}=\frac{2 B_{4} A_{8} A_{9}}{m_{3}^{2} B_{1}-m_{3} B_{2}+B_{25}} \text {, } \\
& B_{38}=\frac{2 B_{4} A_{7} A_{9}}{m_{4}^{2} B_{1}-m_{4} B_{2}+B_{25}} \text {, } \\
& B_{39}=\frac{2 B_{4} A_{5} A_{11}}{m_{5}^{2} B_{1}-m_{5} B_{2}+B_{25}} \text {, } \\
& B_{40}=\frac{2 B_{4} A_{5} A_{12}}{m_{6}^{2} B_{1}-m_{6} B_{2}+B_{25}} \text {, } \\
& B_{41}=\frac{B_{4} A_{9}^{2}}{B_{25}} \text {, } \\
& B_{42}=\frac{1}{\left(e^{m_{11}}-e^{m_{12}}\right)}\left(B_{26}\left(e^{m_{11}}-e^{2 m_{3}}\right)\right. \\
& +B_{27}\left(e^{m_{11}}-e^{2 m_{4}}\right)+B_{28}\left(e^{m_{11}}-e^{\left(m_{4}+m_{3}\right)}\right) \\
& +B_{29}\left(e^{m_{11}}-e^{\left(m_{1}+m_{3}\right)}\right)+B_{30}\left(e^{m_{11}}-e^{\left(m_{1}+m_{4}\right)}\right) \\
& +B_{31}\left(e^{m_{11}}-e^{\left(m_{2}+m_{3}\right)}\right) \\
& +B_{32}\left(e^{m_{11}}-e^{\left(m_{4}+m_{2}\right)}\right)+B_{33}\left(e^{m_{11}}-e^{\left(m_{1}+m_{5}\right)}\right) \\
& +B_{34}\left(e^{m_{11}}-e^{\left(m_{1}+m_{6}\right)}\right) \\
& +B_{35}\left(e^{m_{11}}-e^{\left(m_{2}+m_{5}\right)}\right) B_{36}\left(e^{m_{11}}-e^{\left(m_{6}+m_{2}\right)}\right) \\
& +B_{37}\left(e^{m_{11}}-e^{m_{3}}\right)+B_{38}\left(e^{m_{11}}-e^{m_{4}}\right) \\
& \left.+B_{39}\left(e^{m_{11}}-e^{m_{5}}\right)+B_{40}\left(e^{m_{11}}-e^{m_{6}}\right)+B_{41}\left(e^{m_{11}}-1\right)\right) ; \\
& B_{43}=-\left(B_{41}+B_{42}+B_{26}+B_{27}+B_{28}+B_{29}+B_{31}+B_{30}\right. \\
& \left.+B_{32}+B_{34}+B_{33}+B_{35}+B_{36}+B_{38}+B_{37}+B_{39}+B_{40}\right) \text {. }
\end{aligned}
$$

\section{Data Availability}

The raw data supporting the conclusions of this article will be made available by the authors without undue reservation.

\section{Conflicts of Interest}

The authors declare that they have no conflicts of interest.

\section{Acknowledgments}

The authors are grateful to the Research and Development Wing, Live4Research, Tiruppur, Tamilnadu, India, for the valuable support throughout this project work.

\section{References}

[1] S. Choi, "Enhancing thermal conductivity of fluids with nanoparticles," in Proceedings of the ASME International Mechanical Engineering Congress \& Exposition, vol. 105, no. 66, pp. 99-105, San Francisco, CA, USA, January 1995.

[2] K. S. Nisar, U. Khan, A. Zaib, I. Khan, and A. Morsy, "A novel study of radioactive flow involving micropolar nanoliquid from a shrinking/stretching curved surface including blood gold nanoparticles," The European Physical Journal Plus, vol. 135, no. 10, pp. 1-19, 2020.

[3] M. Sheikholeslami, M. Gorji-Bandpy, D. D. Ganji, and S. Soleimani, "Effect of a magnetic field on natural convection in an inclined half-annulus enclosure filled with $\mathrm{Cu}$-water nanofluid using CVFEM," Advanced Powder Technology, vol. 24, no. 6, pp. 980-991, 2013.

[4] A. S. Dogonchi, M. Alizadeh, and D. D. Ganji, "Investigation of MHD Go-water nanofluid flow and heat transfer in a porous channel in the presence of thermal radiation effect," Advanced Powder Technology, vol. 28, no. 7, pp. 1815-1825, 2017.

[5] K. Loganathan and S. Rajan, "An entropy approach of Williamson nanofluid flow with Joule heating and zero nanoparticle mass flux," Journal of Thermal Analysis and Calorimetry, vol. 141, no. 6, pp. 2599-2612, 2020.

[6] S. Anitha, K. Loganathan, and M. Pichumani, "Approaches for modelling of industrial energy systems: correlation of heat transfer characteristics between magnetohydrodynamics hybrid nanofluids and performance analysis of industrial lengthscale heat exchanger," Journal of Thermal Analysis and Calorimetry, vol. 144, no. 5, pp. 1783-1798, 2020.

[7] A. V. Kuznetsov and D. A. Nield, "Natural convective boundary-layer flow of a nanofluid past a vertical plate," International Journal of Thermal Sciences, vol. 49, no. 2, pp. 243-247, 2010.

[8] W. A. Khan and I. Pop, "Boundary-layer flow of a nanofluid past a stretching sheet," International Journal of Heat and Mass Transfer, vol. 53, no. 11-12, pp. 2477-2483, 2010.

[9] M. H. Abolbashari, N. Freidoonimehr, F. Nazari, and M. M. Rashidi, "Analytical modeling of entropy generation for Casson nano-fluid flow induced by a stretching surface," Advanced Powder Technology, vol. 26, no. 2, pp. 542-552, 2015.

[10] N. A. Sheikh, D. L. C. Ching, I. Khan, D. Kumar, and K. S. Nisar, "A new model of fractional Casson fluid based on generalized Fick's and Fourier's laws together with heat and mass transfer," Alexandria Engineering Journal, vol. 59, no. 5, pp. 2865-2876, 2020.

[11] L. A. Lund, Z. Omar, I. Khan, D. Baleanu, and K. S. Nisar, "Dual similarity solutions of MHD stagnation point flow of Casson fluid with the effect of thermal radiation and viscous dissipation: stability analysis," Scientific Reports, vol. 10, no. 1, pp. 1-13, 2020.

[12] A. Tassaddiq, I. Khan, K. Sooppy Nisar, and J. Singh, "MHD flow of a generalized Casson fluid with Newtonian heating: a fractional model with Mittag-Leffler memory," Alexandria Engineering Journal, vol. 59, no. 5, pp. 3049-3059, 2020. 
[13] S. Nadeem, R. Ul Haq, and C. Lee, "MHD flow of a Casson fluid over an exponentially shrinking sheet," Scientia Iranica, vol. 19, no. 6, pp. 1550-1553, 2012.

[14] C. K. Kumar, S. Srinivas, and A. S. Reddy, "MHD pulsating flow of Casson nanofluid in a vertical porous space with thermal radiation and Joule heating," Journal of Mechanics, vol. 36 , no. 4 , pp. $1-15,2020$.

[15] J. A. Falede and S. O. Adensanya, "MHD oscillatory flow through porous channel saturated with porous media," Alexandria Engineering Journal, vol. 56, pp. 147-152, 2017.

[16] K. Dwivedi, R. K. Khare, and A. Paul, "MHD flow through a vertical channel with porous medium," International Journal of Applied Engineering Research, vol. 13, pp. 11923-11926, 2018.

[17] K. Loganathan, N. Alessa, K. Tamilvanan, and F. S. Alshammari, "Significances of Darcy-Forchheimer porous medium in third-grade nanofluid flow with entropy features," The European Physical Journal Special Topics, 2021.

[18] S. Srinivas, C. K. Kumar, C. K. Kumar, and A. S. Reddy, "Pulsating flow of Casson fluid in a porous channel with thermal radiation, chemical reaction and applied magnetic field," Nonlinear Analysis: Modelling and Control, vol. 23, no. 2, pp. 213-233, 2018.

[19] T. Anwar, P. Kumam, D. Baleanu, I. Khan, and P. Thounthong, "Radiative heat transfer enhancement in MHD porous channel flow of an Oldroyd-B fluid under generalized boundary conditions," Physica Scripta, vol. 95, no. 11, Article ID 115211, 2020.

[20] K. Loganathan, G. Muhiuddin, A. M. Alanazi, F. S. Alshammari, B. M. Alqurashi, and S. Rajan, "Entropy optimization of third-grade nanofluid slip flow embedded in a porous sheet with zero mass flux and a non-fourier heat flux model," Frontiers in Physics, vol. 8, p. 250, 2020.

[21] N. Acharya, K. Das, and P. K. Kundu, "Framing the effects of solar radiation on magneto-hydrodynamics bioconvection nanofluid flow in presence of gyrotactic microorganisms," Journal of Molecular Liquids, vol. 222, pp. 28-37, 2016.

[22] T. Hayat, T. Muhammad, A. Alsaedi, and M. S. Alhuthali, "Magnetohydrodynamic three-dimensional flow of viscoelastic nanofluid in the presence of nonlinear thermal radiation," Journal of Magnetism and Magnetic Materials, vol. 385, pp. 222-229, 2015.

[23] K. Loganathan, K. Mohana, M. Mohanraj, P. Sakthivel, and S. Rajan, "Impact of third-grade nanofluid flow across a convective surface in the presence of inclined Lorentz force: an approach to entropy optimization," Journal of Thermal Analysis and Calorimetry, vol. 144, no. 5, pp. 1935-1947, 2020.

[24] A. Anjum, N. A. Mir, M. Farooq, M. I. Khan, and T. Hayat, "Influence of thermal stratification and slip conditions on stagnation point flow towards variable thicked riga plate," Results in Physics, vol. 9, pp. 1021-1030, 2018.

[25] R. Ahmad, A. Farooqi, J. Zhang, and N. Ali, "Steady flow of a power law fluid through a tapered non-symmetric stenotic tube," Applied Mathematics and Nonlinear Sciences, vol. 4, no. 1, pp. 255-266, 2019.

[26] G. Bary, P. Ru, and W.-N. Zhang, "Analyses of multi-pion Hanbury Brown-Twiss correlations for the pion-emitting sources with Bose-Einstein condensation," Journal of Physics G: Nuclear and Particle Physics, vol. 45, no. 6, 2018.

[27] G. Bary, P. Ru, and W.-N. Zhang, "Normalized multi-pion Hanbury-Brown-Twiss correlation functions of pion-emitting sources with Bose-Einstein condensation," Journal of Physics G: Nuclear and Particle Physics, vol. 46, no. 11, 115107 pages, 2019.
[28] G. M. Sobamowo, "Perturbation methods to analysis of thermal, fluid flow and dynamics behaviours of engineering systems," Perturbation Theory, Intechopen, London, UK, 2020.

[29] E. Elanchezhian, R. Nirmalkumar, M. Balamurugan, K. Mohana, K. M. Prabu, and A. Viloria, "Heat and mass transmission of an Oldroyd-B nanofluid flow through a stratified medium with swimming of motile gyrotactic microorganisms and nanoparticles," Journal of Thermal Analysis and Calorimetry, vol. 141, no. 6, pp. 2613-2623, 2020. 